\title{
Polyphenolic extract from Artemisia selengensis Turcz ameliorates experimental oral mucositis
}

\author{
Fahu Yuan ${ }^{1,2}$, Li Liu², Wenxuan $\mathrm{Yu}^{2}$, Beibei Zhu ${ }^{2}$, Siyuan Sun ${ }^{2}$, Yanbing Jiang ${ }^{2}$, De Chen ${ }^{2}$, and Changli Zeng ${ }^{1, *}$ \\ ${ }^{1}$ Hubei Engineering Research Center for Protection and Utilization of Special Biological Resources in the Hanjiang River Basin, \\ Jianghan University, Wuhan 430056, China \\ ${ }^{2}$ School of medicine, Jianghan University, Wuhan 430056, China
}

\begin{abstract}
Oral mucositis is a common and frequentoccurring disease and there is no effective treatment. In this study, we investigated the efficacy of polyphenolic extract from Artemisia selengensis Turcz (PPAST) in the prevention and treatment of oral mucositis. Cultured human gingival fibroblast cell HGF-1 was used as an in vitro experimental model to confirm the effect of PPAST inhibition of lipopolysaccharide(LPS) on cytotoxicity and its effect on the production of inflammatory cytokines. A rat model of oral ulcer was induced by acetic acid cauterization, and the curative effect of PPAST on oral ulcer was investigated from ulcer area and ulcer duration. PPAST significantly inhibited the toxic effect of LPS on HGF1 cells, improved the survival rate of HGF1 cells, and showed a concentration-dependent inhibition of TNF- $\alpha$ in HGF-1 cell. Treatment with PPAST reduced mucositis scores, promoted oral ulcer healing, and reduced plasma TNF- $\alpha$ levels in rats. Experimental data show that PPAST is safe and effective in the prevention and treatment of oral inflammatory diseases.
\end{abstract}

\section{Introduction}

Oral inflammatory diseases are common and frequentlyoccurring diseases. Almost everyone has the experience of suffering from oral inflammatory diseases, such as Oral mucositis, oral ulcers, periodontitis, gingivitis and periodontitis. Although oral inflammatory diseases are self-healing to a certain extent, oral inflammation can cause discomfort, swelling, pain or bleeding, and seriously affect work and life. Some patients even suffer from pain or bleeding, which may affect their eating or language, lead to dyspepsia, mental anxiety and anxiety, and greatly reduce their quality of life[1].

In oral inflammatory diseases, periodontal inflammation refers to the inflammation of periodontal tissues such as gum, periodontal membrane and alveolar bone. The initial manifestations are gingivitis symptoms such as gingival bleeding and gingival swelling and pain, and periodontitis symptoms such as periodontal pocket, gingival atrophy and tooth loosening after the aggravation of inflammation. The main pathogenic bacteria of periodontal inflammation is gram-negative anaerobe, and the endotoxin lipopolysaccharide (LPS) in the cell wall is the main pathogenic factor of periodontal inflammation. Human gingival fibroblast (HGFs) is the main cell in the gingival connective tissue and plays an important role in the protection and repair of periodontal tissue[2]. In the pathological process of periodontal inflammation, LPS has obvious cytotoxic effect on gingival fibroblasts, the main cell of human periodontal tissue, inhibiting the growth of gingival fibroblasts, cell proliferation and DNA synthesis. LPS stimulates macrophages, monocytes, fibroblasts and periodontal tissue cells to produce and secrete various inflammatory mediators, such as PGE 2, IL-1 $\beta$, TNF- $\alpha$ and IL-6. Under the action of inflammatory mediators, it causes enhanced vascular permeability and leukocyte infiltration, inhibits collagen synthesis, and causes gingival bleeding and gingival swelling and pain[3].

Artemisia selengensis Turcz is a famous herb in China. It is recorded in both the Classic of Materia Medica and Compendium of Materia Medica. Artemisia selengensis Turcz is widely distributed in China and has been used since ancient times to reduce fever, dysentery and acute and chronic hepatitis. Previous studies have found that the pharmacological action of Artemisia selengensis Turcz is closely related to its active components such as phenolic acid, flavonoids and alkaloids[4]. Our previous study found that the polyphenolic extract from Artemisia selengensis Turcz(PPAST) could inhibit the proliferation of many pathogenic bacteria.

The purpose of this study was to investigate the effect of PPAST on the prevention and treatment of oral mucositis by anti-inflammatory and promoting the healing of oral ulcers. With cultured human gingival fibroblasts as the experimental model, Cell Counting Kit-8 was used to detect the inhibitory effect of Artemisia quinoa extract and Artemisia quinoa extract on gingival fibroblast toxicity, and the effect

* Corresponding author: zengchangli6111@ @ohu.com 
concentration was preliminarily screened. Then the effects of artemisia selengensis extract and Artemisia selengensis extract on the production of IL-1 $\beta$, TNF- $\alpha$ and IL- 6 by LPS stimulation to gingival fibroblasts cell HGF-1 were detected by Elisa. In SD rats, the oral ulcer model was established by acetic acid cauterization. The efficacy of PPAST were investigated from ulcer diameter, ulcer area and ulcer duration.

\section{Material and method}

\subsection{Preparation of polyphenolic extract from Artemisia selengensis Turcz}

The leaves of Artemisia selengensis Turcz were dried at $60{ }^{\circ} \mathrm{C}$ and crushed, and every 20 gram of Artemisia selengensis Turcz leaves were added with $300 \mathrm{~mL} \mathrm{75 \%}$ ethanol extract solution, mixed, treated by ultrasonic wave for 35 minutes, stood for 20 minutes, centrifuged at 5000 RPM for 15 minutes, the supernatant was taken, concentrated by rotary evaporation and freeze-dried, and the PPAST obtained was stored in the dryer for use.

\subsection{Cell culture}

Human gingival fibroblasts cellline HGF-1 in the logarithmic growth stage were selected for culture. The cells were digested and collected by trypsin, and then added into high-sugar DMEM medium containing 20\% (volume fraction) fetal bovine serum to make cell suspension, and inoculated on 96-well cell culture plate or 6-well cell culture plate. Cells were cultured in an incubator of $\mathrm{CO}_{2}$ with a volume fraction of $5 \%$ at $37{ }^{\circ} \mathrm{C}$.

\subsection{Cell proliferation and cytotoxicity assay}

Using the Cell Counting Kit-8(purchased from Beyotime Biotechnology Co., LTD., China), a rapid, highsensitivity assay based on WST- 8 and widely used in cell proliferation and cytotoxicity detection, cells was divided into three groups: LPS-induced inflammation group: LPS $(20 \mu \mathrm{g} / \mathrm{mL})$; PPAST treatment group $(0.01$ $\mu \mathrm{g} / \mathrm{mL}, 0.1 \mu \mathrm{g} / \mathrm{mL}$, and $1 \mu \mathrm{g} / \mathrm{mL})+\operatorname{LPS}(20 \mu \mathrm{g} / \mathrm{mL})$; negative control group: only cells and culture medium. The logarithmic phase cells were collected and seeded into 96-well plate, placed in the cell culture tank for overnight culture, and the drugs were added according to the above groups, and continued incubation and culture for $24 \mathrm{~h}$, according to the operating instructions, $10 \mu \mathrm{L}$ CCK-8 solution was added to each well, and the OD450 was read after incubation at $37{ }^{\circ} \mathrm{C}$ in dark for 30 minutes.

\subsection{Quantitative determination of cytokines}

Monolabelled cells were cultured overnight, and the culture medium was abandoned the next day. After washing each well with PBS for 3 times, $200 \mu \mathrm{L}$ of DMEM containing different concentrations of PPAST and LPS were added to each well, respectively. After 24 hours of culture, the concentrations of IL-1, IL-6 and TNF- $\alpha$ in the supernatant of the above cultures were detected by ELISA.

\subsection{Rat model of oral ulcer}

Thirty-two SD rats were selected for experimental study, 8 rats were randomly selected as the normal control group, and the remaining 24 rats were prepared with oral ulcer rat model by acetic acid burning method. After the success of the model, the rats were randomly divided into model control group and PPAST treatment group, with 8 rats in each group. The normal control group and model control group were given normal feeding without drug treatment. In the PPAST treatment group, $1 \mathrm{~mL}$ of PPAST suspension was applied with a sterile cotton swab, once per day, and administered continuously for 7 d. During the period, observed the change of oral mucosa, determination of no swelling mucosa ulcer area and observe the inflammatory hyperemia, and digital camera photograph ulcer change records, observation in a day before giving treatment, using vernier caliper measured ulcer diameter, and high definition digital camera, with ImageJ software for grey value, and calculate the area of ulcer, oral ulcer building success to completely heal time of ulcer healing time.

On the 8th day, the rats were sacrificed, and the oral mucosal tissues were fixed with formalin, and then paraffin-embedded, followed by $\mathrm{H} \& \mathrm{E}$ staining. With optical microscope check each slide ulcer site inflammatory cell infiltration, epithelial and the change of the tube and score according to the following grades: score 0 : no or without erythema and normal mucosa hyperemia, no bleeding area, ulcer, or abscess; score 1 : moderate erythema and congestion, no bleeding area, ulcer or abscess; score 2: severe erythema and hyperemia, bleeding area, small ulcers or scar tissue, but no abscess; score 3: severe erythema and congestion, hemorrhage region of the existence of a wide range of ulcer and mucosal abscess.

\subsection{Statistical analysis}

The experimental data is expressed as the mean \pm standard deviation, and SPSS 13.0 is used to perform a one-way analysis of variance on the experimental data. $P<0.05$ is considered as a significant difference.

\section{Results}

\subsection{PPAST alleviates the effects of LPS-induced human gingival fibroblast toxicity}

LPS showed significant cytotoxicity on human gingival fibroblasts, with a growth inhibition rate of $40.5 \%$. However, PPAST with a concentration of $0.01 \sim 0.1$ $\mu \mathrm{g} / \mathrm{mL}$ inhibited the cytotoxicity of LPS, and the cell survival rate of PPAST was $73.5 \%$, when the concentration was $0.01 \mu \mathrm{g} / \mathrm{mL}$ (Fig.1). 


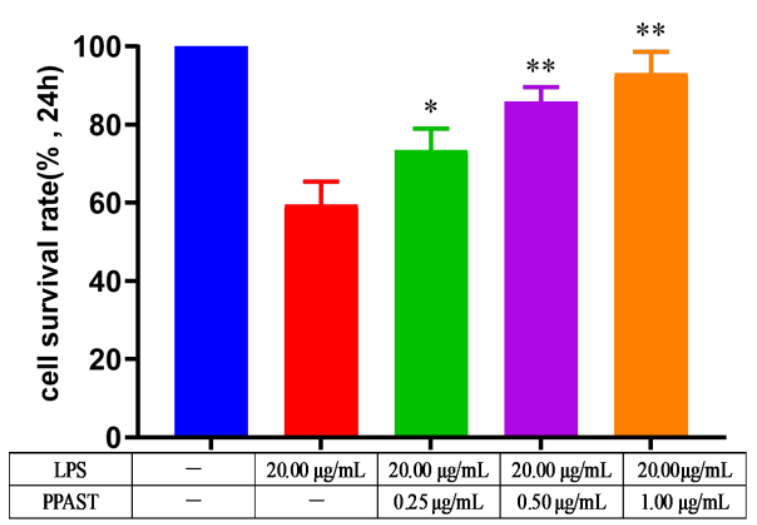

Fig. 1. PPAST alleviates the growth inhibition of HGF-1 by LPS. $* \mathrm{P}<0.05, * * \mathrm{P}<0.01$ compared with modle group.

\subsection{PPAST inhibited the production of IL-1 $\beta$, TNF- $\alpha$ and IL- 6 in HGF-1 induced by LPS}

After $24 \mathrm{~h}$ of culture, there were statistically significant differences in the levels of inflammatory factors in the supernatant of cell culture medium in each group. The levels of IL- $1 \beta$, IL- 6 and TNF- $\alpha$ in the model group (treated with LPS only) were significantly higher than those in the blank control group $(P<0.01)$. The levels of the above three inflammatory cytokines in PPAST treatment were all lower than those in the model control group $(P<0.01)$, andand there was a dose-dependent relationship (Fig. 2).

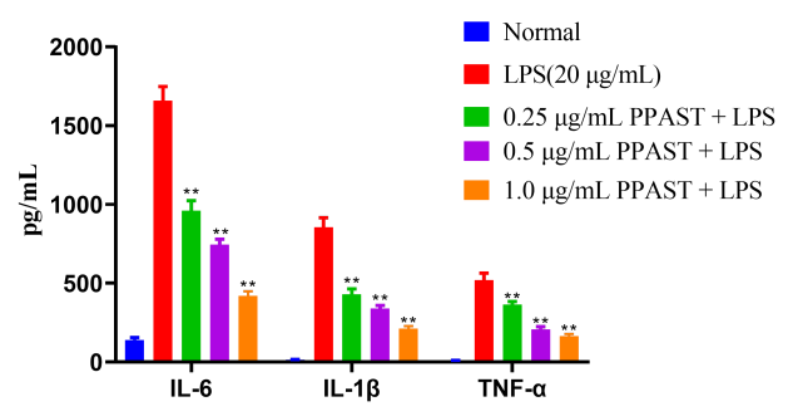

Fig. 2. PPAST inhibits the expression of inflammatory factors in HGF-1 induced by LPS. $* * \mathrm{P}<0.01$ compared with modle group.

\subsection{PPAST improves oral ulcers in rats}

When the ulcer model was established, elliptic bleeding surface of oral ulcer and a few brown burns could be seen. On the 3rd day, the ulcer had the largest area and was elliptic, covered by yellow-white fibrous membrane, and the surrounding mucosa was accompanied by erythema halo. On the 6th day, the center of the ulcer was a yellow-white pseudomembrane mucosa, with obvious congestion and edema in the surrounding mucosa, and the ulcer area decreased. On the 9th $\sim 12$ th day, the ulcer area was gradually reduced to healing and mucosal hyperemia and edema were also gradually alleviated(Fig. 3 ).
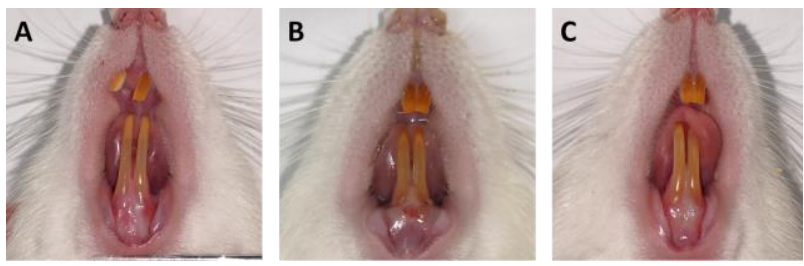

Fig. 3. Treatment with PPAST significantly reduced the symptoms of gingival mucosal ulcer in rats after 4 days. A normal control group, B: oral mucositis modle group, C: PPAST treatment group.

The experimental results of the effect of PPAST on oral ulcer in rats are shown in table 1 and figure 4 . Before administration, there were no significant differences in the diameter and area of oral ulcer in rats in the three experimental groups $(P>0.05)$. From day 1 to day 4 after administration, the ulcer diameter and ulcer area of rats in the PPAST group were significantly smaller than those in the corresponding oral ulcer model group $(\mathrm{P}<0.05)$. The ulcer healing time of PPAST group was significantly shorter than that of the oral ulcer model group $(P<0.05)$.

Table 1. Effect of PPAST on oral ulcer area in SD rats induced by acetic acid $(\mathrm{n}=8$, mean $\pm \mathrm{SD}) . * \mathrm{P}<0.05,{ }^{*} * \mathrm{P}<0.01$ compared with modle group.

\begin{tabular}{|c|c|c|c|c|c|c|}
\hline \multirow{2}{*}{ Group } & \multicolumn{6}{|c|}{ Ulcer area $\left(\mathrm{mm}^{2}\right)$} \\
\cline { 2 - 7 } & day 0 & day 1 & day 2 & day 3 & day 4 & day 5 \\
\hline Oral & $43.08 \pm$ & $46.11 \pm$ & $49.38 \pm$ & $40.08 \pm$ & $33.71 \pm 5$ & $30.78 \pm$ \\
mucositis & 4.55 & 5.29 & 4.55 & 6.11 & .02 & 5.14 \\
modle & & & & & & \\
\hline PPAST & $43.87 \pm$ & $44.67 \pm$ & $44.07 \pm$ & $37.76 \pm$ & $28.47 \pm 4$ & $26.12 \pm$ \\
treatment & 4.06 & 4.87 & $4.38^{*}$ & $4.22^{*}$ & $.27 * *$ & $3.95^{*}$ \\
\hline
\end{tabular}

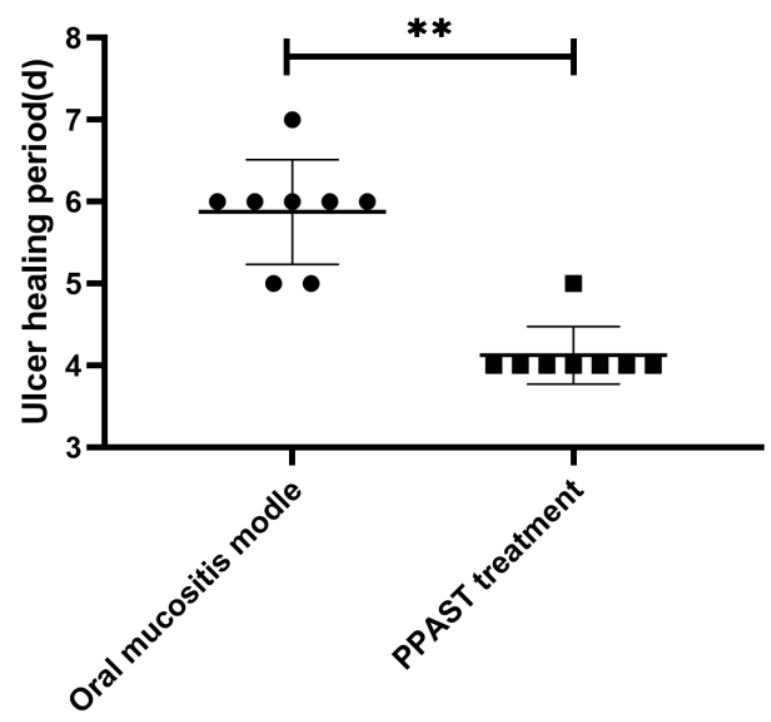

Fig. 4. PPAST shortened the healing time of oral ulcers in SD rats induced by acetic acid. $* * \mathrm{P}<0.01$ compared with modle group.

The results of H\&E staining showed that the keratinization layer, granule layer, spinous layer and basal layer of oral mucosa of rats in the normal group were intact and clear, and there were fewer lymphocytes, 
erythrocyte extravasation and tissue cell infiltration. In the local tissue of the model group, the epithelium was damaged and shed, the layer of spinous cells was loose, and a large number of inflammatory cells were infiltrated. In the PPAST group, compared with the model group, inflammatory cell infiltration was reduced, and the mucosal tissue structure became complete and clear.

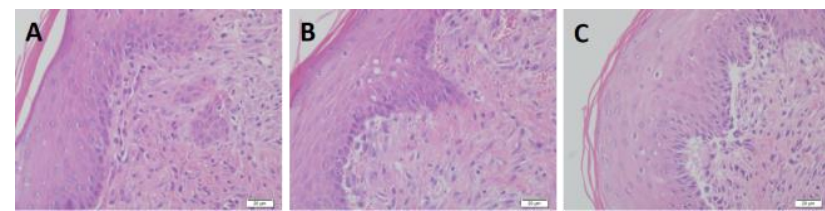

Fig. 5. A: normal control group, B: oral mucositis modle group, C: PPAST treatment group.

\section{Discussion}

This study is the first to explore the effect of PPAST on oral mucositis from the perspective of tissue inflammation and healing. The results showed that, compared with the control group, PPAST increased the ulcer healing area by $16 \% \sim 37 \%$ at the early stage of ulcer, especially at the early stage of ulcer (D2 D4), with histological features of reduced inflammatory cells, increased collagen fiber content, and accelerated reepithelialization. However, at the late stage of ulcer (D6 $\sim$ D7), PPAST had no significant effect on ulcer area and other histological indicators, except for the number of inflammatory cells. These findings suggest that PPAST can improve the healing process of oral mucositis at the early stage of ulcer, and may be related to inhibiting inflammation and promoting tissue repair.

The development process of ulcer includes three stages: inflammation initiation and ulcer formation, inflammation diffusion and ulcer exacerbation, inflammation regression and tissue healing. In the process from inflammation initiation to inflammation diffusion, the gradually increasing inflammation has the bactericidal effect, but it also causes some unnecessary tissue destruction. Subsequently, the inflammation was gradually reduced and the tissue also turned to repair and healing. The healing process of oral mucositis in this study was consistent with this trend. From d2 to d4, inflammatory cells were significantly increased, but the collagen fiber content was not significantly changed, which may be in the stage of inflammation diffusion and ulcer aggravation. From d4 to d7, inflammatory cells were significantly reduced, collagen fiber content and degree of re-epithelialization were increased, consistent with the characteristics of inflammation regression and tissue healing[5].

The improvement of collagen fiber content and reepithelialization by PPAST in the late oral mucositis stage is not obvious, which may be related to the doubleside of inflammation. The process of tissue repair is closely related to inflammation. Destructive inflammation leads to the aggravation of tissue damage and hindering epithelial regeneration, but late restorative inflammation can promote tissue and epithelial repair. It suggests that the anti-inflammatory effect of PPAST on tissue regeneration may also have two sides, that is, early inhibition of inflammation can reduce tissue destruction caused by inflammation, but later inhibition of inflammation also inhibits tissue regeneration.

\section{Conclusion}

In conclusion, PPAST has a significant improvement effect on oral mucositis, especially in the early stage of ulcer, which may be related to inhibiting inflammation and promoting tissue repair.

\section{Acknowledgments}

This project is funded by the Engineering Technology Research Center for the Protection, Development and Utilization of Characteristic biological Resources in The Hanjiang River Basin of Hubei Province. This paper is one of the phased results of the open subject project (2019-06) of the Engineering Technology Research Center for the Protection, Development and Utilization of Characteristic biological resources in the Hanjiang River Basin of Hubei Province.

\section{References}

1. Wardill Hannah R., Tissing Wim J E., Kissow Hannelouise., Stringer Andrea M.(2019). Animal models of mucositis: critical tools for advancing pathobiological understanding and identifying therapeutic targets. Curr Opin Support Palliat Care, 13(2), 119-133.

2. Anturlikar Suryakanth Dattatreya., Azeemuddin Mohammed Mukhram., Varma Sandeep., Mallappa Onkaramurthy., Niranjan Dilip., Krishnaiah Ashok Basti., Hegde Shruthi Manjunath., Rafiq Mohamed., Paramesh Rangesh.(2019). Turmeric based oral rinse "HTOR-091516" ameliorates experimental oral mucositis. Ayu, 40(2), 127-133.

3. Kato Takao., Segami Natsuki., Sakagami Hiroshi.(2016). Anti-inflammatory Activity of Hangeshashinto in IL-1 $\beta$-stimulated Gingival and Periodontal Ligament Fibroblasts. In Vivo, 30(3), 257-63.

4. Shi Feng., Jia Xiaobin., Zhao Chenglei., Chen Yan.(2010). Antioxidant activities of various extracts from Artemisisa selengensis Turcz (LuHao). Molecules, 15(7), 4934-46.

5. Harada Koji., Ferdous Tarannum., Mizukami Yoichi., Mishima Katsuaki.(2018). Elemental diet inhibits pro-inflammatory cytokine production in keratinocytes through the suppression of NF- $\kappa \mathrm{B}$ activation. Oncol. Rep., 40(1), 361-368. 